\title{
Etude théorique de la conduction bidimensionnelle instationnaire dans un mur soumis à un flux radiatif uniforme
}

\author{
F. Yguel $(*)$ et F. Penot $(* *)$ \\ (*) Laboratoire d'Energétique Solaire. \\ $\left({ }^{* *}\right)$ Laboratoire d'Etudes Aérodynamiques et Thermiques, 40, avenue du Recteur Pineau, 86022 Poitiers cedex, \\ France
}

(Reçu le 8 juillet 1981, révisé le 30 septembre 1981, accepté le 7 octobre 1981)

\begin{abstract}
Résumé. - Cette étude concerne la résolution numérique de l'équation de la conduction de la chaleur dans un mur soumis sur une face à une densité de flux radiatif uniforme et, de part et d'autre, à des conditions aux limites de convection naturelle et de rayonnement. Les trois régimes de convection naturelle sont pris en compte pour la détermination du coefficient d'échange local sur les parois. On détermine le profil de température, les puissances convectées, conduites et rayonnées sur chacune des faces, en fonction des cinq paramètres adimensionnels qui caractérisent le problème.

On montre que les variations longitudinales du flux conduit, justifient l'emploi d'un schéma à deux dimensions.
\end{abstract}

\begin{abstract}
This study deals with numerical resolution of the heat conduction equation in a thick vertical wall submitted on one side to an uniform heat flux density, and to convective and radiative boundaries conditions on both sides. The three regimes of natural convection are taken into consideration for the determination of the local heat transfer coefficient.

We determines the temperature-profile, the convective-radiative and conductive out puts on each face; versus the five dimensionless parameters concerned with that problem.

It is shown that the conduction effects justifie the use of a bidimensional scheme.
\end{abstract}

\section{Notations.}

a Diffusivité thermique de l'air

$B_{i}(y, t)=\frac{h_{i}^{*}\left(y^{*}, t^{*}\right) l}{\lambda_{\mathrm{s}}}=$ Nombre de Biot

$C \quad$ Chaleur spécifique du mur

$1 / d=H / l$ Allongement du mur

$g \quad$ Accélération de la pesanteur

$G r_{y}=\frac{g \beta\left(T^{*}-T_{0}^{*}\right)}{v^{2}} y^{* 3}$ Nombre de Grashof local,

fonction de $t$ et $y$

$G r_{0}=\frac{g \beta \theta_{\mathrm{c}} H^{3}}{v^{2}}$ Nombre de Grashof caractéristique

$l \quad$ Epaisseur du mur

$H \quad$ Hauteur du mur

$h_{i}^{*}\left(y^{*}, t^{*}\right)$ Coefficient local de convection

$N u_{y} \quad$ Nombre de Nusselt local, fonction de $t$

$P=\frac{\varepsilon_{1} \sigma T_{0}^{* 4}}{\tau Q_{\mathrm{s}}}$ Paramètre adimensionnel

$\operatorname{Pr}=v / a$ Nombre de Prapndtl

$Q_{\mathrm{s}} \quad$ Densité de flux radiatif incidente

$Q_{\text {conv }} \quad$ Densité de flux convecté
$Q_{\text {cond }} \quad$ Densité de flux conduit

$Q_{\text {ray }} \quad$ Densité de flux rayonné

$T_{0}^{*} \quad$ Température ambiante

$T^{*}\left(x^{*}, y^{*}, t^{*}\right)$ Température locale

$T=\frac{T^{*}-T_{0}^{*}}{\theta_{\mathrm{c}}}$ Température adimensionnelle

$T_{1}=T_{0}^{*} / \theta_{\mathrm{c}}$ Température ambiante adimensionnelle

$T_{\mathrm{m}} \quad$ Température moyenne adimensionnelle du mur

$t^{*} \quad$ Temps

$t_{\mathrm{c}}=l^{2} / \alpha$ Temps de Fourier caractéristique

$t=t^{*} / t_{\mathrm{c}} \quad$ Temps adimensionnel

$x^{*}, y^{*} \quad$ Variables d'espace

$x=x^{*} / l, y=y^{*} / H$ Variables d'espaces adimensionnelles

\section{Alphabet grec}

$\alpha \quad$ Diffusivité thermique du solide

$\rho_{\mathrm{s}} \quad$ Masse volumique du mur

$\varepsilon_{1}, \varepsilon_{2} \quad$ Emissivités des faces $x=0$ et $x=1$, respectivement

$\varepsilon=\varepsilon_{2} / \varepsilon_{1}$ Paramètre adimensionnel

$\lambda_{\mathrm{a}} \quad$ Conductivité thermique de l'air 
$\lambda_{\mathrm{s}} \quad$ Conductivité thermique du mur

$\lambda=\lambda_{\mathrm{a}} / \lambda_{\mathrm{s}}$ Paramètre adimensionnel

$\theta_{\mathrm{c}}=l \tau Q_{\mathrm{s}} / \lambda_{\mathrm{s}}$ Ecart de température caractéristique

$\tau \quad$ Coefficient d'absorption du mur

$\sigma \quad$ Constante de Stefan

$v \quad$ Viscosité cinématique de l'air

$i=1 \quad$ sur la face $x=0$

$i=2 \quad$ sur la face $x=1$

1. Introduction. - Le problème de la conduction bidimensionnelle dans un mur vertical, soumis sur une face à une densité de flux uniforme, et à des conditions aux limites de convection naturelle et de rayonnement, n'a pas reçu, jusqu'ici, de solution complète. En effet, la majorité des travaux sur le couplage conduction-convection-rayonnement est relative à la convection forcée [1], [2], [3], [4], et le rayonnement y est souvent négligé [1], [3]. La formulation du problème est d'ailleurs identique que l'on se place en convection naturelle ou forcée, si on utilise un coefficient d'échange constant à la paroi. Cette dernière hypothèse autorise l'emploi de modèles monodimensionnels.

Lorsque les échanges convectifs ne sont plus pris en compte à travers une valeur unique, deux cas distincts ont, à notre connaissance, été envisagés dans la littérature :

- quelques auteurs ont considéré un coefficient d'échange constant dans le temps, mais variant spatialement le long du mur [6], [7].

- d'autres auteurs ont résolu le problème instationnaire, mais ces coefficients d'échange sont conservés constants dans l'espace [2], [4], [5], [8].

Dans tous ces travaux, les échanges radiatifs ont été modélisés à l'aide de la loi de Stefan [2], [4], [8], ou ont été le plus souvent négligés.

Si l'on s'intéresse aux échanges thermiques d'un mur d'épaisseur finie soumis brusquement à un rayonnement unilatéral (en l'absence d'écoulement extérieur forcé) les considérations d'ordre physique qui suivent nous imposent de tenir compte d'au moins deux dimensions spatiales, et de l'évolution temporelle des phénomènes.

En effet, dans les premiers instants, la face soumise au rayonnement va s'échauffer, entraînant la formation d'une couche limite de convection naturelle. La conduction s'installe, provoque l'échauffement de la face opposée où se développe alors la seconde couche limite de convection naturelle. Les structures des deux couches limites seront d'autant plus différentes que la résistance thermique du mur sera importante. En particulier, les zones de transition laminaire-turbulent seront situées à des cotes distinctes sur les deux faces. Le déphasage thermique introduit ainsi une dissymétrie aussi bien spatiale que temporelle dans l'évolution des transferts thermiques tant radiatifs que convectifs. Il est important de noter, qu'à chaque instant, la solution dépend de l'historique du phénomène depuis l'instant initial.

La présente étude traite donc le problème conjugué complet, avec des coefficients d'échanges variables dans l'espace et le temps, dans le cas du mur bidimensionnel. Elle présente en outre, l'originalité de prendre en compte les trois régimes de convection naturelle, ne limitant pas ainsi l'étude aux murs de faible hauteur (régime laminaire uniquement). Cette approche trouve des applications nombreuses, notamment en thermique du bâtiment.

2. Equations et conditions aux limites. - On considère ici un mur homogène de grande largeur et d'épaisseur finie, placé dans une atmosphère à température constante $T_{0}^{*}$. Une des faces est soumise à une densité de flux constante $Q_{s}$. Les faces horizontales sont supposées adiabatiques (Fig. 1).

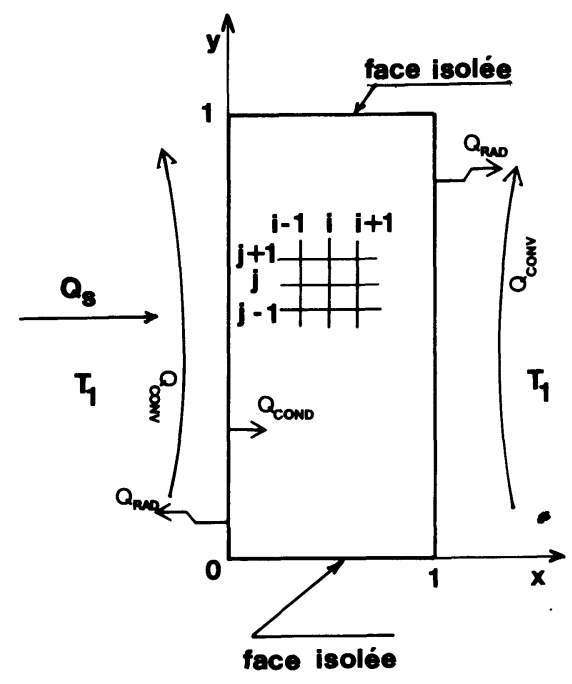

Fig. 1. - Conditions aux limites du problème.

[Boundaries conditions for the problem.]

Les échanges thermiques au niveau des faces verticales $x^{*}=0$ et $x^{*}=l$ se font par rayonnement et convection naturelle avec leur environnement à la température $T_{0}^{*}$.

En utilisant les notations de la figure 1, on définit le flux de chaleur $Q_{\text {conv }}$ cédé à l'atmosphère ambiante par convection naturelle, par :

$$
Q_{\text {conv }}=h_{i}^{*}\left(y^{*}, t^{*}\right)\left(T^{*}-T_{0}^{*}\right)
$$

de même, le flux de chaleur rayonné par le mur vers l'extérieur s'écrit :

$$
Q_{\text {ray }}=\varepsilon_{i} \sigma\left(T^{* 4}-T_{0}^{* 4}\right)
$$

le flux transféré transversalement par conduction locale à l'intérieur du mur a pour expression :

$$
Q_{\text {cond }}=-\lambda_{s} \frac{\partial T^{*}}{\partial x^{*}} .
$$


On suppose qu'à l'instant initial on soumet la face $x^{*}=0$ du mur à un flux $Q_{\mathrm{s}}$ constant, le mur étant à l'équilibre thermique avec son environnement. Le bilan instantané de quantité de chaleur sur les deux faces verticales du mur s'écrit respectivement, quel que soit $t>0$ :

$$
\begin{gathered}
\text { - pour } x^{*}=0, \quad 0<y^{*}<H \\
Q_{\text {cond }}=\tau Q_{\mathrm{s}}-Q_{\mathrm{ray}}-Q_{\mathrm{conv}} \\
\text { - pour } x^{*}=l, 0<y^{*}<H \\
Q_{\mathrm{cond}}=Q_{\mathrm{ray}}+Q_{\mathrm{conv}} .
\end{gathered}
$$

L'échauffement instantané du mur est formulé par l'équation bidimensionnelle de la conduction de la chaleur :

- pour $0<x^{*}<l, 0<y^{*}<H, t^{*}>0$

$$
\lambda_{s}\left(\frac{\partial^{2} T^{*}}{\partial x^{* 2}}+\frac{\partial^{2} T^{*}}{\partial y^{* 2}}\right)=\rho_{\mathrm{s}} C \frac{\partial T^{*}}{\partial t^{*}}
$$

Les quatre angles du mur sont des points singuliers, où les conditions aux limites s'écrivent :

$$
\begin{aligned}
& \text { - } x^{*}=0, y^{*}=0 \text { et } y^{*}=H \\
& -\lambda_{\mathrm{s}} \frac{\partial T^{*}}{\partial x^{*}}+\gamma \lambda_{\mathrm{s}} \frac{\partial T^{*}}{\partial y^{*}}=\tau Q_{\mathrm{s}}-\varepsilon_{1} \sigma\left(T^{* 4}-T_{0}^{* 4}\right)- \\
& -h_{1}^{*}\left(y^{*}, t^{*}\right)\left(T^{*}-T_{0}^{*}\right) \\
& \text { - } x^{*}=l, y^{*}=0 \text { et } y^{*}=H \\
& -\lambda_{\mathrm{s}} \frac{\partial T^{*}}{\partial x^{*}}+\gamma \lambda_{\mathrm{s}} \frac{\partial T^{*}}{\partial y^{*}}=\varepsilon_{2} \sigma\left(T^{* 4}-T_{0}^{* 4}\right)+ \\
& +h_{2}^{*}\left(y^{*}, t^{*}\right)\left(T^{*}-T_{0}^{*}\right)
\end{aligned}
$$

dans lesquelles $\gamma$ vaut 1 ou -1 , lorsque $y^{*}$ est respectivement égal à 0 ou $H$.

Les faces horizontales sont supposées adiabatiques, on a donc :

- pour $y^{*}=0$ ou $y^{*}=H, 0<x^{*}<l$

$$
-\lambda_{\mathrm{s}} \frac{\partial T^{*}}{\partial y^{*}}=0
$$

Dans cette étude, nous avons supposé que $Q_{\mathrm{s}}^{\cdot}$ ne dépend pas du temps. Ceci nous permet de définir un écart de température $\theta_{c}$, caractéristique de la conduction à travers un mur d'épaisseur $l$, de conductivité thermique $\lambda_{\mathrm{s}}$ et soumis à un flux $\tau Q_{\mathrm{s}}\left(\theta_{\mathrm{c}}=l \tau Q_{\mathrm{s}} / \lambda_{\mathrm{s}}\right)$. La température adimensionnelle $T$, s'écrit alors comme le rapport entre l'écart de température, $T^{*}-T_{0}^{*}$, et $\theta_{c}$ : elle permet ainsi, bien qu'il s'agisse d'un problème de conduction de tenir compte de la présence du phénomène convectif.

Si on définit de plus les variables réduites $x=x^{*} / l$ $y=y^{*} / H$ et $t=t^{*}\left(\alpha / l^{2}\right)$, les équations (6), (7), (8) et (9) s'écrivent alors sous forme adimensionnelle :
- $0<x<1,0<y<1$

$$
\frac{\partial^{2} T}{\partial x^{2}}+d^{2} \frac{\partial^{2} T}{\partial y^{2}}=\frac{\partial T}{\partial \eta}
$$

où $1 / d$ représente l'allongement du mur.

$$
\text { - } x=0,0<y<1
$$

$$
\begin{gathered}
-\frac{\partial T}{\partial x}=1+P\left[1-\left(\frac{T}{T_{1}}+1\right)^{4}\right\rceil-B_{1}(y, t) T \\
-x=1,0<y<1 \\
-\frac{\partial T}{\partial x}=\varepsilon P\left[\left(\frac{T}{T_{1}}+1\right)^{4}-1\right]+B_{2}(y, t) T
\end{gathered}
$$

dans lesquelles, $B_{1}$ et $B_{2}$ sont des nombres de Biot, $P$ et $\varepsilon P$, des paramètres sans dimension, qui caractérisent respectivement, les échanges convectifs et radiatifs sur les deux faces.

Une formulation sans dimension analogue a été obtenue pour les quatre points singuliers.

- $y=0$ et $y=1,0<x<1$

$$
-\frac{\partial T}{\partial y}=0 \text {. }
$$

3. Paramètres sans dimension. - Les équations (10) à (13) montrent que le problème dépend de six paramètres adimensionnels : $B_{1}, B_{2}, P, T_{1}, d$ et $\varepsilon$.

Etant donné que les nombres de Biot $B_{1}$ et $B_{2}$ sont liés au nombre de Nusselt par les relations

- pour $y \neq 0$

$$
B_{i}(y, t)=\frac{h_{i}^{*}\left(y^{*} t^{*}\right) l}{\lambda_{\mathrm{s}}}=\frac{N u_{y, t}}{y} \lambda d
$$

- pour $y=0$

$$
B_{i}(y, t)=0
$$

et que ce dernier est, par ailleurs, une fonction du nombre de Grashof local $\operatorname{Gr}(y)$ pour chaque zone de convection considérée, si on pose

$$
G r_{y, t}=G r_{0} T y^{3}
$$

le problème se trouve alors défini par les six paramètres adimensionnels suivants : $\lambda d, G r_{0}, P, T_{1}, d$ et $\varepsilon$.

Les calculs que nous avons effectués, montrent en fait l'indépendance des résultats vis-à-vis de $d$, si $d$ reste inférieur ou égal à 0,3 . En effet, dans ces cas, les variations de $d$ ne provoquent que des modifications négligeables des températures adimensionnelles locales et moyennes. En particulier pour $\lambda d=4 \times 10^{-4}$; $G r_{0}=3 \times 10^{11} ; P=0,3 ; T_{1}=3$ et $\varepsilon=1$; où le problème risque d'être fortement bidimensionnel, l'accroissement de $d$ de 0,05 à 0,3 n'entraîne que des écarts maximums de $3 \%$ sur les températures adimensionnelles locales et $3 \%$ sur les températures moyennes. 
Ainsi, si on se limite à des murs de grand allongement $(d<0,3)$, le problème est complètement défini par les cinq paramètres $\lambda d, G r_{0}, P, T_{1}$ et $\varepsilon$. Les effets longitudinaux sont principalement liés aux conditions aux limites différentes sur les faces verticales.

De plus, si l'on effectue l'hypothèse des faibles écarts de température, c'est-à-dire si $T / T$; $\ll 1$, le problème dépend seulement des quatre paramètres $\lambda d, G r_{0}$, $P / T_{1}$ et $\varepsilon$. Cette hypothèse, qui se trouve vérifiée si $\theta_{c}$ est inférieur à $100 \mathrm{~K}$, ne sera pas retenue dans le cadre de cet article.

4. Choix des coefficients d'échange convectif. Etant donné les faibles variations de la température le long des faces (cf. $\S 6$ ), les coefficients d'échange convectif ont été déterminés à partir des relations empiriques $\left(G r_{y}, N u_{y}\right)$ établies à température de paroi constante, soit :

pour la zone laminaire (9), où $G r_{v}<1,04 \times 10^{9}$

$$
N u_{y}=\left(0,358+0,3877 \cdot\left(G r_{y}\right)^{-1 / 2}\right) \cdot\left(G r_{v}\right)^{1 / 4}
$$

pour la zone turbulente (10), où $G r_{y}>1,06 \times 10^{10}$

$$
N u_{y}=0,115 \cdot\left(G r_{y}\right)^{1 / 3}
$$

pour la zone transition (11), où :

$$
\begin{gathered}
\begin{array}{c}
1,04 \times 10^{9}<G r_{y}<1,06 \times 10^{10} \\
N u_{y}=C_{1} \log ^{5} Z+C_{2} \log ^{4} Z+C_{3} \log ^{3} Z+ \\
+C_{4} \log ^{2} Z+C_{5} \log Z+C_{6}
\end{array} \\
\text { où }: \begin{aligned}
Z=G r_{y} & \\
C_{1}=-1,27 & C_{4}=1,40 \times 10^{3} \\
C_{2}=91,37 & C_{5}=4,26 \times 10^{5} \\
C_{3}=-1,99 \times 10^{3} & C_{6}=3,70 \times 10^{6} .
\end{aligned}
\end{gathered}
$$

Cette dernière relation a été tirée d'une étude expérimentale de la transition sur une plaque plane verticale maintenue à une température constante, supérieure de $57 \mathrm{~K}$ à la température ambiante [10], [11].

On pourra noter (Fig. 2) l'existence de discontinuités en début et en fin de zone de transition. Bien que plus complexe qu'une fonction linéaire reliant la fin de la zone laminaire au début de la zone turbulente établie, la relation (18) rend compte des variations importantes du nombre de Nusselt dans la zone de transition. Des essais effectués en remplaçant (18) par une loi linéaire conduisent à des écarts de l'ordre de $15 \%$ sur les flux convectés.

5. Méthode de résolution numérique. - L'équation de la conduction de la chaleur (10) et les conditions aux limites associées (11-13) ont été résolues à l'aide d'un schéma numérique aux directions alternées (A.D.I.) [12], l'organigramme général de la méthode est présenté sur la figure 3.

Dans l'évolution au cours du temps, les conditions aux limites font intervenir la température locale de paroi et les nombres de Biot $B_{i}(y, t)$ qui sont inconnus. La non-linéarité résultant du terme de rayonnement

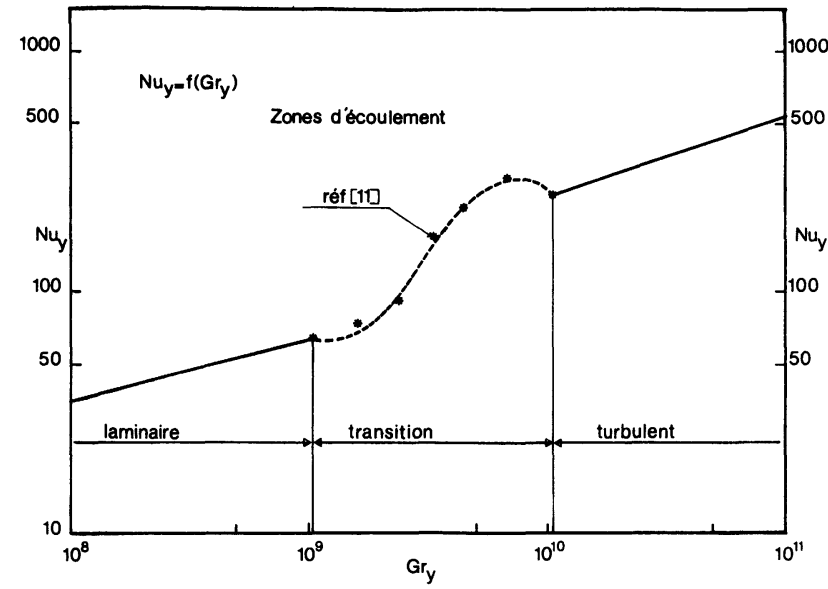

Fig. 2. - Evolution du nombre de Nusselt en fonction du nombre de Grashof pour chacune des 3 zones de convection.

[Evolution of Nusselt number, versus Grashof number for the three regimes of natural convection.]

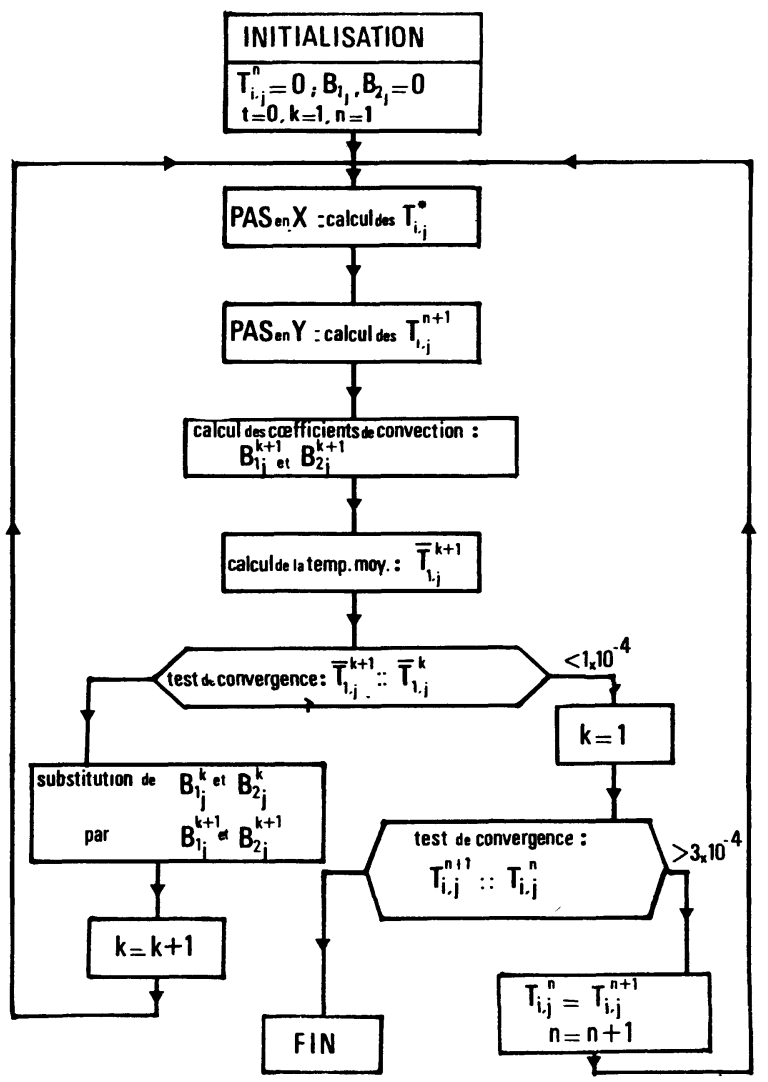

Fig. 3. - Organigramme de la méthode de résolution numérique.

[Flowchart of the numerical resolution algorithm.]

est traitée aux deux demi-pas de temps du schéma (A.D.I.) en posant :

$$
\begin{aligned}
\left(\frac{T^{n+1 / 2}}{T_{1}}+1\right)^{4}=\left(\frac{T^{n}}{T_{1}}+1\right)^{4} & +4\left(\frac{T^{n}}{T_{1}}+1\right)^{3} \\
& \times \frac{T^{n+1 / 2}-T^{n}}{T_{1}}
\end{aligned}
$$




$$
\begin{aligned}
\left(\frac{T^{n+1}}{T_{1}}+1\right)^{4}=\left(\frac{T^{n+1 / 2}}{T_{1}}\right. & +1)^{4}+4\left(\frac{T^{n+1 / 2}}{T_{1}}+1\right)^{3} \\
& \times \frac{T^{n+1}-T^{n+1 / 2}}{T_{1}} .
\end{aligned}
$$

Celle provenant du produit $B_{i}(y, t) T$ est supprimée en introduisant un schéma itératif sur $B_{i}(y, t)$ qui utilise au départ les valeurs du nombre de Biot au pas de temps précédent. Le test de convergence du processus itératif est effectué sur la température moyenne de la face $x=0$. Le calcul montre qu'une valeur relative de $10^{-4}$ entre deux itérations successives est suffisante pour assurer la progression d'un pas de temps.

De manière à intégrer le phénomène de choc thermique au temps $t=0$, deux pas de temps ont été utilisés : un pas de temps adimensionnel de $5 \times 10^{-3}$ a été choisi pour les 50 premières itérations, un pas de temps double est ensuite employé.

Le maillage utilisé comporte 101 nœuds suivant $y$ et 11 suivant $x$. Il a été vérifié numériquement que la diminution des pas d'espaces en $x$ et $y$ n'apporte que des modifications négligeables du profil des différents flux, excepté dans les zones de début et de fin de transition, où l'influence des points anguleux de la relation $\left(G r_{y}, N u_{y}\right)$ devient plus ponctuelle. Les profils de température, par contre, ne subissent que des variations infimes.

6. Résultats, discussion. - Tel qu'il est formulé, le problème de la conduction bidimensionnelle instationnaire fait intervenir de nombreux paramètres $\left(\lambda d, G r_{0}, P, T_{1}\right.$ et $\left.\varepsilon\right)$ qui sont associés à une évolution spatiale et temporelle des phénomènes.

Les résultats sont présentés suivant trois niveaux distincts. Sur un exemple précis, avec des valeurs fixes des paramètres, nous discutons les solutions locales qui donnent à différents instants le champ de température dans le mur et la répartition des flux de chaleur conduits, convectés et rayonnés.

On indique de même, comment ce calcul permet de déterminer à chaque instant les caractéristiques moyennes du mur telles que sa température, ou les températures moyennes de paroi.

Enfin nous effectuons une étude de sensibilité de paramètre en considérant les modifications apportées aux transferts conductifs, convectifs et radiatifs pris à un instant donné.

6.1 EVolution De La température et DU FluX THERMIQUE DANS LE MUR. - On traite ici d'un cas particulier communément rencontré en thermique du bâtiment, et pour lequel les conclusions peuvent être généralisées $\left(\lambda d=6 \times 10^{-4}, G r_{0}=5,6 \times 10^{11}\right.$, $\left.P=0,3, T_{1}=3, \varepsilon=1\right)$.

La figure 4 donne la répartition de température du mur à $t=0,25$ et $t=1$. Les écarts de température enregistrés sur les faces $x=0$ et $x=1$, traduisent les différences de transfert convectif entre chacune des

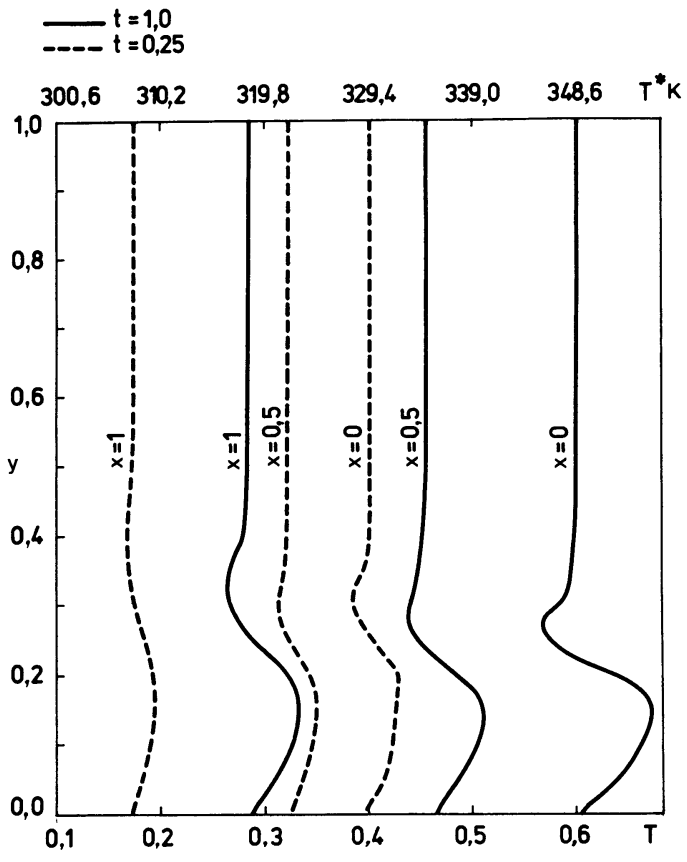

Fig. 4. - Profils de température suivant $x, y$ et $t$ pour : $\lambda d=6 \times 10^{-4}, G r_{0}=5,6 \times 10^{11}, P=0,3, T_{1}=3$ et $\varepsilon=1$.

[Temperatures profiles with $x, y$ and $t$ for : $\lambda d=6 \times 10^{-4}$, $G r_{0}=5.6 \times 10^{11}, P=0.3, T_{1}=3, \varepsilon=1$.

trois zones de convection. Ces écarts augmentent en fonction du temps en même temps que le coefficient d'échange convectif.

L'échelle dimensionnée de la figure 4 , relative au cas particulier : $l=0,1 \mathrm{~m} ; H=3,5 \mathrm{~m} ; \lambda_{\mathrm{s}}=1,25 \mathrm{Wm}^{-1}$ $\mathrm{K}^{-1} ; \tau Q_{\mathrm{s}}=1200 \mathrm{Wm}^{-2} ; \mathrm{T}_{0}=291 \mathrm{~K}$, montre cependant, qu'à chaque instant, les écarts de température sur chacune des parois ne sont que de quelques degrés Kelvin seulement.

Les variations du flux conduit sur les parois $x=0$ et $x=1$ (Fig. 5), sont par contre plus importantes

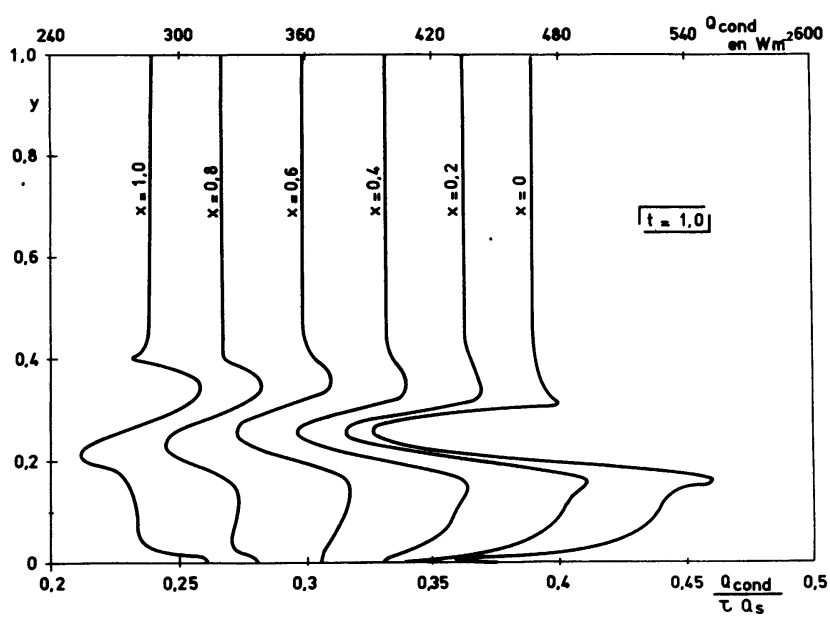

Fig. 5. - Distribution du flux conduit dans le mur $\left(\lambda d=6 \times 10^{-4}, G r_{0}=5,6 \times 10^{11}, P=0,3, T_{1}=3, \varepsilon=1\right)$.

[Evolution of the conductive flux in the wall $\left(\lambda d=6 \times 10^{-4}\right.$, $\left.G r_{0}=5.6 \times 10^{11}, P=0.3, T_{1}=3, \varepsilon=1\right)$. $]$ 
(l'échelle dimensionnée de la figure 5 se réfère aux mêmes valeurs qu'à la figure 4). Le profil des flux conduit et convecté, résulte en effet des variations suivant $y$, de la température de paroi et du coefficient d'échange $B_{i}$, celles-ci s'amplifiant avec le temps. Ce résultat permet de justifier l'emploi de relations empiriques entre les nombres de Grashof et de Nusselt à température de paroi constante, plutôt qu'à flux constant.

On notera que les éventuels sauts de flux présents sur les courbes et qui proviennent des points anguleux de la relation $\left(G r_{y}, N u_{y}\right)$ n'affectent qu'une région infime du profil. Ils ne sauraient mettre en doute la validité globale des résultats.

La figure 6 présente le bilan du transfert thermique à la solution stationnaire. Des modifications importantes du flux de chaleur par conduction dans le mur subsistent encore.

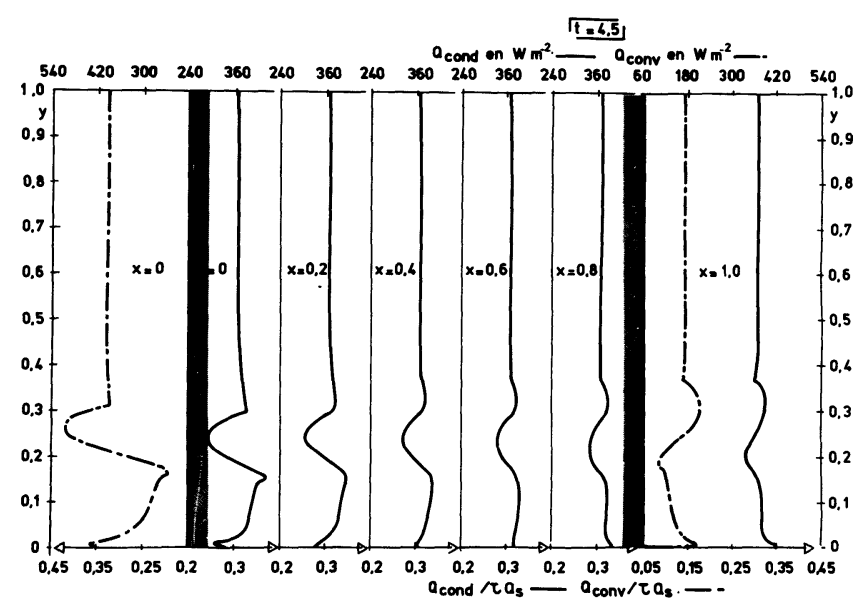

Fig. 6. - Représentation suivant $x$ et $y$ des puissances convectées et conduites à la solution stationnaire (cas identique à celui de la figure 4).

[Sketch of steady state convective and conductive fluxes, as a function of $x$ and $y$ (parameters identical to those of figure 4).]

Du fait d'un niveau de température différent sur les deux faces verticales, les hauteurs de transition laminaire-turbulent ne sont pas les mêmes et par suite, à une cote donnée, les transferts convectifs ne sont pas identiques. La conduction dans le sens vertical vient alors compenser ces variations non négligeables. Cette conduction apparaît comme un élément essentiel du transfert de chaleur dans le mur. Elle ne peut en conséquence être négligée, et justifie l'emploi d'un modèle numérique à deux dimensions.

6.2 Caractéristiques globales DU MUR. - Si l'on se réfère toujours au même exemple, l'" histoire " du transfert de chaleur dans le mur peut être établie en considérant l'évolution dans le temps des moyennes spatiales des flux transmis par conduction, convection et rayonnement. Sous une forme adimensionnelle ceux-ci s'écrivent respectivement $\overline{Q_{\text {cond }} / \tau Q_{s}}, \overline{Q_{\text {conv }} / \tau Q_{s}}$,

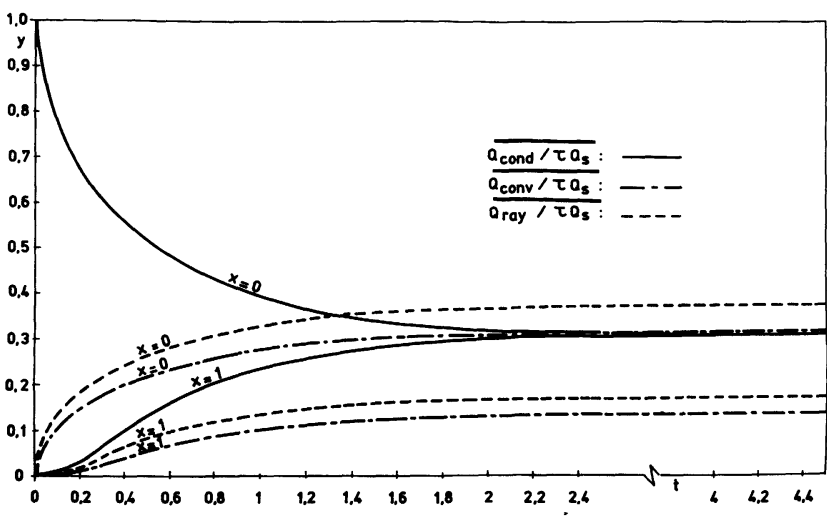

Fig. 7. - Variation dans le temps des puissances conduites, convectées et rayonnées sur chacune des faces (cas identique à celui de la figure 4).

[Evolution in time of convective, conductive and radiative outputs on each face (parameters identical to those of figure 4).]

$\overline{Q_{\text {ray }} / \tau Q_{s}}$. Ils sont définis de manière identique par $\int_{0}^{1} \frac{Q(x, y, t)}{\tau Q_{\mathrm{s}}} \mathrm{d} y$. Ils sont représentés figure 7 sur les deux faces $x=0$ et $x=1$. On remarque que dans les premiers instants, seule la conduction sur la face soumise au rayonnement est importante. Le mur commençant à se réchauffer, les transferts radiatifs et convectifs apparaissent d'abord sur la face $x=0$, puis sur la face $x=1$. La conduction dans le mur introduit un déphasage entre le transfert sur les deux faces. Plus tard, le système tend vers un état stationnaire, le mur atteint une température d'équilibre non uniforme, les flux conduits sur les deux faces sont alors égaux.

A chaque instant, on vérifie bien que sur la face insolée, le flux transmis par conduction est le complémentaire des flux emportés par convection et rayonnement, tandis que sur la face opposée, le flux conduit se transforme en convection et rayonnement.

La figure 8 montre l'évolution dans le temps de la

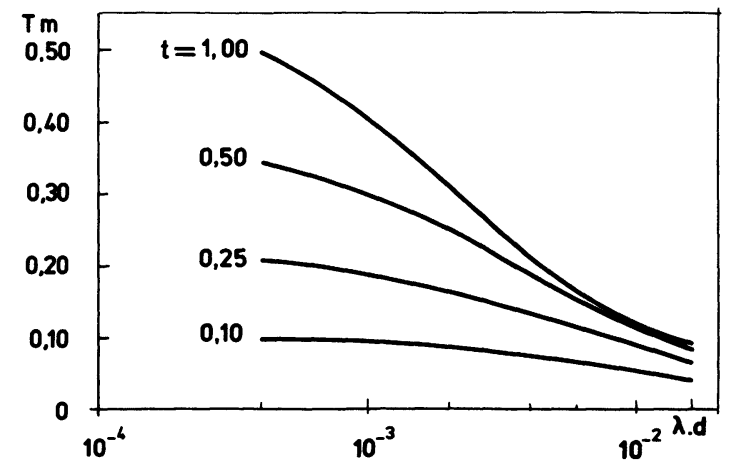

Fig. 8. - Variations de la température moyenne du mur en fonction de $\lambda d$ et à différentes valeurs de $t\left(G r_{0}=3 \times 10^{11}\right.$, $P=0,3, \varepsilon=1$ ).

[Mean wall temperature evolution, versus $\lambda d$, at different times $\left(G r_{0}=3 \times 10^{11}, P=0.3, \varepsilon=1\right)$.] 
température moyenne du mur $T_{\mathrm{m}}$, définie par $\int_{0}^{1} \int_{0}^{1} T(x, y) \mathrm{d} x \mathrm{~d} y$. Celle-ci est une mesure de l'énergie thermique accumulée. On note que la température d'équilibre du mur est d'autant plus grande que $\lambda d$ est petit. Ceci résulte de la définition de la température adimensionnelle $T$ qui croît avec $\lambda_{\mathrm{s}}$ et $1 / l$ (et en conséquence avec $1 / \lambda d)$. Cette température d'équilibre est atteinte pour un temps d'autant plus long que $\lambda d$ est petit.

6.3 MODIFICATIONS DES CARACTÉRISTIQUES GLOBALES DU MUR. - Si l'on considère le système d'équations sans dimensions (10)-(13) on doit s'attendre à trouver que :

- L'augmentation de $P$ provoque la réduction des flux conduit et convecté au profit du flux rayonné (Eqs. (11-12)). $P$ est en effet représentatif de la part respective du rayonnement émis par l'environnement du mur, vis-à-vis du rayonnement incident $Q_{\mathrm{s}}$.

- La diminution de $T_{1}$ induit de même une réduction des flux conduits et convectés (cf. $\S 3$ ).

- Le paramètre $G r_{0}$ qui exprime le niveau de turbulence, tend à faire croître, avec lui, les échanges convectifs.

- La décroissance de $\varepsilon$ entraîne l'augmentation de la température d'équilibre, de même que celle des échanges convectifs.

Ces remarques sur l'évolution des phénomènes, relativement à la variation d'un paramètre, ne sont que qualitatives. Seuls des réseaux de courbes du type de ceux présentés aux figures 9 et 10 permettent de caractériser quantitativement l'influence de l'ensemble des groupements adimensionnels.

On définit un nombre de Biot moyen $\bar{B}_{1}$ par $\bar{B}_{1}=\int_{0}^{1} B_{\mathrm{i}}(y, t) \mathrm{d} y$. Les figures $9 a, 9 b, 9 c$ précisent l'évolution de $\bar{B}_{1}$ pris à l'instant $t=0,5$ en fonction de $\dot{\lambda}$. Dans chaque cas de figure, deux paramètres restent fixes et les courbes sont paramétrées suivant la troisième grandeur, respectivement $G r_{0}, P, T_{1}$. Pour faciliter la présentation on a admis que $\varepsilon$ restait égal à l'unité. De la même façon les figures $10 a, 10 b, 10 c$, déterminent les parts respectives des échanges conductifs et radiatifs de la face soumise au rayonnement.

- Variation de $G r_{0}$. - Gr $r_{0}$ est, comme on pouvait s'y attendre, le paramètre dont l'influence est la plus nette sur $\bar{B}_{1}$. Son décuplement fait croître $\bar{B}_{1}$ d'un facteur de 2 à 3 (Fig. $9 a$ ), ce qui se traduit par une diminution du flux conduit de 10 à $15 \%$ environ (Fig. 10a). Pour des valeurs de $\lambda d$ supérieures à $10^{-2}$ le flux radiatif est pratiquement inexistant lorsque $G r_{0}=10^{12}$. Mais celui-ci peut atteindre $40 \%$ pour une valeur identique de $G r_{0}$ si $\lambda d$ devient faible.

- Variation de $T_{1}$. - L'accroissement de $T_{1}$ entraîne l'augmentation du coefficient d'échange convectif (Fig. 9b). Il demeure relativement constant dès que $T_{1}$ devient voisin de 10 . Lorsque $T_{1}$ est faible, le

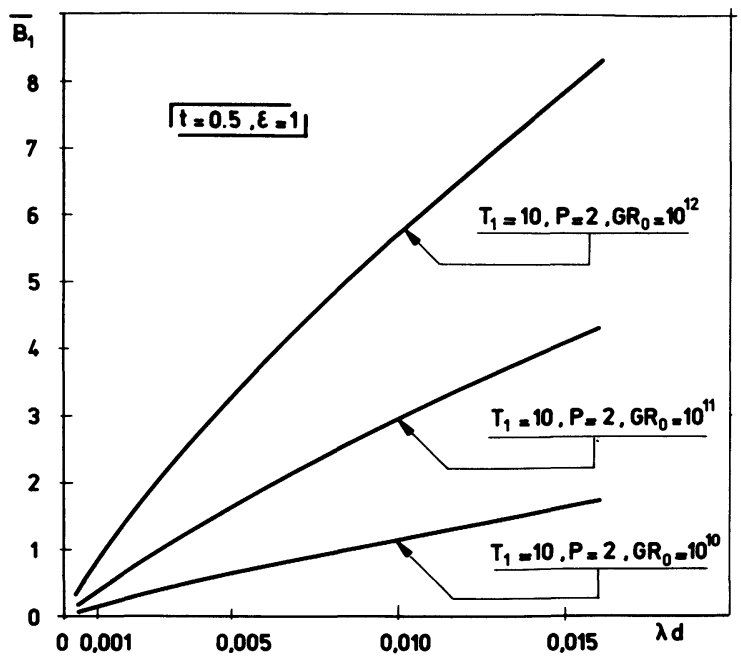

a)

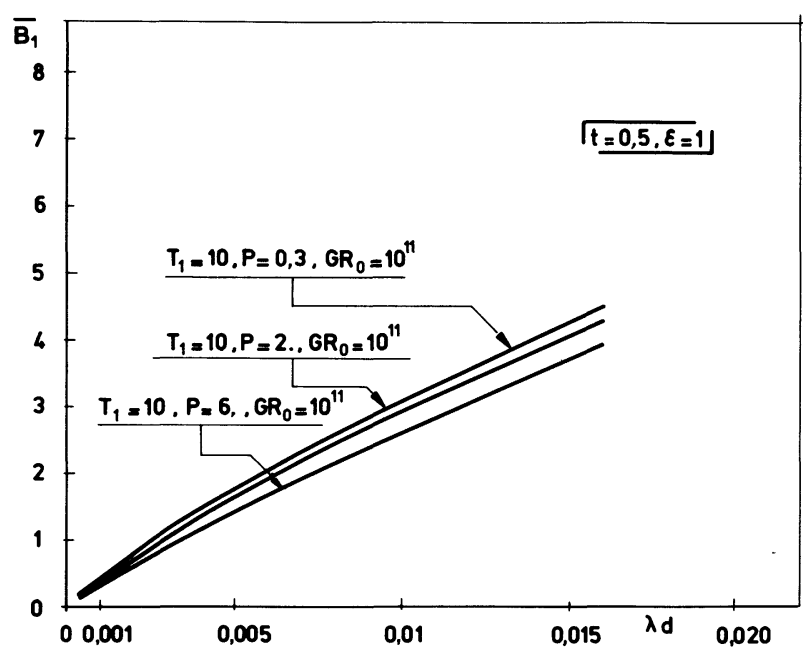

b)

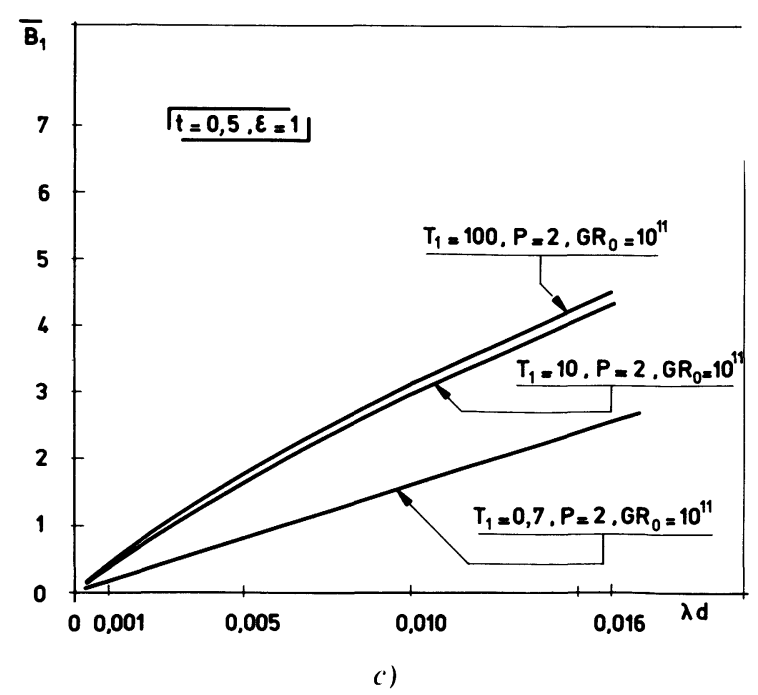

Fig. 9. - $a$ ), b), c). - Evolutions du nombre de Biot en fonction de l'évolution des paramètres du problème $(t=0,5$, $\varepsilon=1$ )

$[a), b), c)$ Biot number evolution as a function of the problem parameters $(t=0.5, \varepsilon=1)$.] 


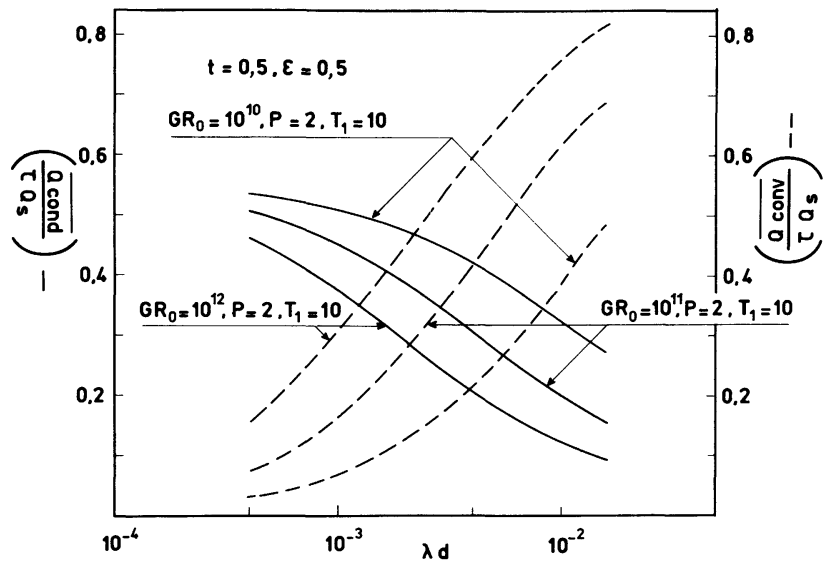

a)

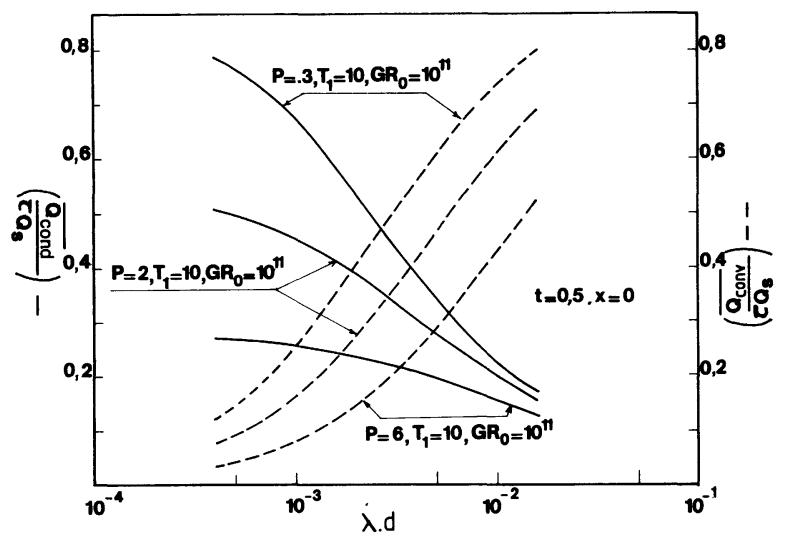

b)

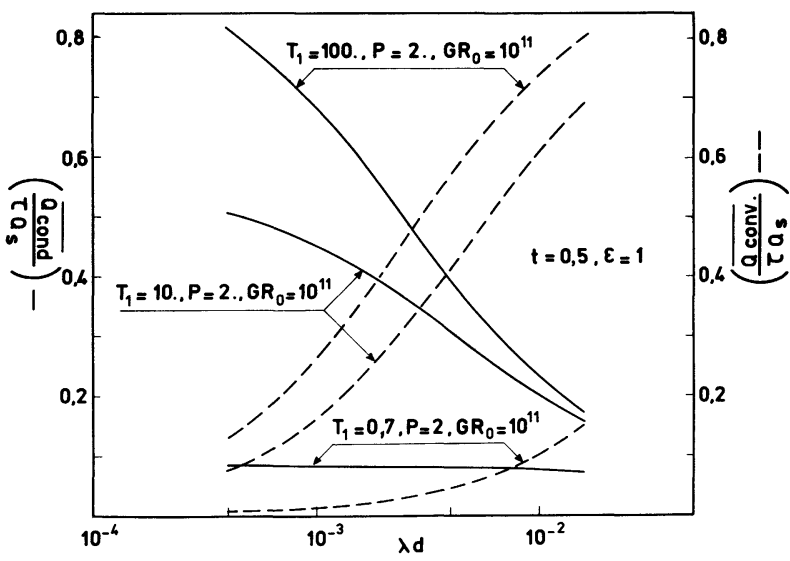

c)

Fig. 10. $-a), b$ ), c) Evolutions des flux conduits et convectés en fonction de l'évolution des paramètres du problème $(t=0,5, \varepsilon=1)$.

$[a), b), c)$ Conductive and convective fluxes, versus the evolution of the problem parameters $(t=0.5, \varepsilon=1)$.] problème est essentiellement radiatif : $75 \%$ à $90 \%$ du flux incident est restitué à l'environnement sous forme de rayonnement, quel que soit $\lambda d$. Lorsque $T_{1}$ croît, et pour des valeurs faibles de $\lambda d$, le rayonnement reste dominant, mais le transfert conductif augmente, pour atteindre $50 \%\left(\lambda d=5 \times 10^{-4}\right)$ lorsque $T_{1}$ vaut 10 . Par contre, lorsque $\lambda d$ est grand, on a affaire à un cas convecto-radiatif où la conduction ne représente plus que $20 \%$ environ du flux incident (Fig. 10b).

- Variation de $P$. - Sur la figure $9 c$, on note que $\bar{B}_{1}$ varie relativement peu avec $P$ (10 à $20 \%$ environ). Contrairement aux cas où $T_{1}$ est petit, les transferts radiatifs peuvent être négligés, indépendamment de $\lambda d$, lorsque $P$ est faible (de l'ordre de 0,3). De faibles valeurs de $\lambda d$, associées à de grandes valeurs de $P$, réduisent ce problème couplé aux seuls échanges par rayonnement et conduction.

Ces différentes remarques montrent que, pour des valeurs moyennes de ces cinq paramètres, aucun transfert de chaleur ne peut être négligé, et qu'il est nécessaire de se référer à chaque fois à des abaques. La difficulté de la présentation vient en effet, que l'on dépasse le cadre élémentaire de la classification habituelle :

- Corps à résistance interne négligeable, auquel se rattachent deux cas $B_{i} \gg 1$, et $B_{i} \ll 1$.

- Plaque épaisse semi-infinie, associée aux deux sous-groupes précédents, qui ne permet de traiter qu'une faible partie de cas pratiques, où certaines simplifications sont possibles.

7. Conclusion. - L'étude présentée apporte une réponse complète au problème bidimensionnel de la conduction instationnaire dans un mur. La prise en compte des trois régimes de convection naturelle permet d'obtenir avec précision la valeur des flux convecté, conduit et rayonné.

Bien que le flux radiatif incident soit constant, les variations verticales de la température de paroi, moins importantes que celles du flux conduit, nous permettent de valider le choix de relations $\left(G r_{y}, N u_{y}\right)$ à température de paroi constante plutôt qu'à flux constant.

Si d'autre part, les faibles variations de température sur les parois semblent rapprocher ce problème de ceux à température constante, généralement monodimensionnels, il apparaît, en fait, que la conduction longitudinale est un élément essentiel du fonctionnement de ce mur. Elle permet d'équilibrer les différences spatiales entre les puissances convectées de part et d'autre du mur, et justifie pleinement l'emploi d'un schéma à deux dimensions.

\section{Bibliographie}

[1] Gosse, J., Analyse du couplage conduction-convection pour un écoulement à couche limite laminaire sur une plaque plane. Communication aux Journées d'Etude sur les Ecoulements Turbulents et les Transferts de Chaleur, C. R. Orsay (1977).
[2] ZYSKOWSKI, W., The transient temperature distribution in a slab subjected to radiative and convective heating calculated by variational method. Int . J. Heat Mass Transf. 16 (1972) 917-932. 
[3] LI'IKov, A. V., Conjugate convective heat transfert problems. Int. J. Heat Mass. Transf. 17 (1973) $257-$ 265.

[4] Milton, J. I., Goss, W. P., On solving the transient conducting slab with radiating and convecting surfaces. J. Heat Trans. (1974) 547-549.

[5] Chang, Y. P., Analytical solution for heat conduction in anisotropie media in infinite, semi-infinite, and two-plane-bounded regions. Int. J. Heat Mass Transf. 20 (1977) 1019-1028.

[6] Kellemer, M. D., YANG, K. T., A steady conjugate heat transfer problem with conduction and free convection. Appl. Sci. Res. 17 (1967) 249-269.

[7] ZINNES, A. E., The coupling of conduction with laminar natural convection from a vertical flat plate with arbitrary surface heating. J. Heat Transf. (1970) 528-535.
[8] Crosbie, A. L. and Viskanta, R., Transient heating on cooling of a plate by combined convection and radiation. Int. Journal Heat Mass Trans. 11 (1968) 305-317.

[9] Riley, D. S., Drake, D. G., Appl. Sci. Res. 30 (1975) 193.

[10] DoAn Kim Son, Contribution à l'étude de la zone de transition et de la zone de turbulence établie dans un écoulement de convection naturelle sur une plaque plane verticale isotherme. Thèse de Docteur-ès-Sciences de l'Université de Poitiers (1977).

[11] DOAN Kim Son, Communication personnelle.

[12] RoAche, P. J., Computational fluid dynamics, Sandialaboratories Albuquerque (Hermosa Publishers) New-Mexico 87115 (1972). 